Research Article

\title{
Invariance of Deficiency Indices of Second-Order Symmetric Linear Difference Equations under Perturbations
}

\author{
Yan Liu (1D \\ College of Science, Hohai University, Changzhou, Jiangsu 213022, China \\ Correspondence should be addressed to Yan Liu; yanliu@hhu.edu.cn
}

Received 19 November 2019; Accepted 19 December 2019; Published 13 February 2020

Academic Editor: Gestur Ólafsson

Copyright (C) 2020 Yan Liu. This is an open access article distributed under the Creative Commons Attribution License, which permits unrestricted use, distribution, and reproduction in any medium, provided the original work is properly cited.

This paper focuses on the invariance of deficiency indices of second-order symmetric linear difference equations under perturbations. By applying the perturbation theory of Hermitian linear relations, the invariance of deficiency indices of the corresponding minimal subspaces under bounded and relatively bounded perturbations is built. As a consequence, the invariance of limit types of second-order symmetric linear difference equations under bounded and relatively bounded perturbations is obtained.

\section{Introduction}

Consider the following second-order difference equation:

$$
-\nabla(p(t) \Delta x(t))+q(t) x(t)=\lambda w(t) x(t), \quad t \in I,
$$

where $I$ is the integer set $\{t\}_{t=0}^{+\infty} ; \Delta$ and $\nabla$ are the forward and backward difference operators, respectively, i.e., $\Delta x(t)=$ $x(t+1)-x(t), \nabla x(t)=x(t)-x(t-1) ; p(t)$ and $q(t)$ are all real-valued with $p(t) \neq 0$ for $t \in I$ and $p(-1) \neq 0 ; w(t)$ is the weight function with $w(t)>0$ for $t \in I$; and $\lambda$ is a complex spectral parameter.

Equation (1) originates from the discretization of the onedimensional Schrödinger equation $[1,2]$. It is the simplest form of formally self-adjoint difference equations. Due to its wide applications (see [3]), equation (1) has been extensively studied, and many elegant results have been obtained. In particular, Atkinson [3] first studied the limit point and limit circle types for second-order difference equations. Subsequently, his work was further developed by Hinton, Jirari, Clark, Chen, et al. (see [4-7]). Later, Sun and Shi $[8,9]$ studied the eigenvalue problems of second-order difference equations with coupled boundary conditions and periodic and antiperiodic boundary conditions, respectively. Tx'hey proved the existence of eigenvalues and calculated the numbers of eigenvalues. Then, by applying the Glazman-Krein-Naimark theory for Hermitian linear relations, self-adjoint extensions for second-order symmetric linear difference equations were completely characterized in terms of boundary conditions [10]. Recently, we studied regular approximations of spectra of singular second-order symmetric linear difference equations. It was shown that spectral inclusion and spectral exactness hold in the limit point and limit circle case, separately [11-13]. For more results about second-order difference equations, the reader is referred to $[14,15]$. In this paper, we are interested in the stability of deficiency indices of the minimal subspaces generated by these singular second-order difference equations under bounded and relatively bounded perturbations.

It is well known that the deficiency indices of closed symmetric operators or Hermitian linear relations play an important role in the theory of self-adjoint extension. According to the generalized von Neumann theory [16] and the GKN theory [17], a closed symmetric operator or a Hermitian linear relation has a self-adjoint extension if and only if its positive and negative deficiency indices are equal and its self-adjoint extension domains have a close relationship with its deficiency indices. So, it is of great significance to determine the deficiency indices of both differential equations and difference equations. No doubt, perturbation theory is one of the important research tools. 
Stability of deficiency indices of differential operators under perturbations has been extensively studied, and many elegant results have been obtained. In 1910, Weyl first studied the deficiency indices of second-order formally self-adjoint differential equations [18]. It was shown that the deficiency index equals to the number of linearly independent square integrable solutions of the differential equation for each spectral parameter $\lambda \in \mathbf{C} / \mathbf{R}$ (where $\mathbf{C}$ and $\mathbf{R}$ denote the sets of the complex and real number, respectively). Later, Atkinson studied the maximal deficiency index of Hamiltonian differential systems [3]. He showed that the maximal deficiency index is invariant under bounded perturbation. In particular, the stability of deficiency indices of symmetric operators under perturbations was studied systematically by Behncke and Focke [19]. They proved the invariance of deficiency index of a symmetric operator under relatively bounded perturbation with relative bound less than 1 . This result extends the well known self-adjointness result [20]. In the relative bound one case, however, the deficiency index may shrink (for more results about the stability of the deficiency indices of symmetric operators, see [21-23] and some references cited therein).

In the study of spectral theory of difference expression (1) as well as the general linear discrete Hamiltonian systems, it was found that the minimal operator may be not densely defined or not well defined as an operator; that is, it is multivalued [13]. This fact is ignored in previous literature, including [24-26]. So, the classical perturbation theory of deficiency indices of symmetric operators is not available in this case. Motivated by the need to consider the operators of this kind, Neumann [27] first introduced linear relations into functional analysis, and then Arens [28] and many other scholars further studied and developed the fundamental theory of linear relations. A linear relation is also called a linear subspace (briefly, subspace). In particular, motivated by the study of the stability of deficiency indices of (1), we generalized the corresponding results in [19] for symmetric operators to Hermitian linear relations and established the perturbation theory of deficiency indices of Hermitian linear relations [29]. In addition, the invariance of self-adjointness of linear relations was obtained in [30]. In this paper, we shall apply these results given in [29] to discuss the stability of deficiency indices of (1) under bounded and relatively bounded perturbations.

To the best of our knowledge, there seem a few results about the stability of the deficiency indices of Hermitian subspaces under perturbations, though there are many results about the deficiency indices of Hermitian relations (see $[3,4,17,26,31-36])$. In 1998, Cross introduced concepts of boundedness, relative boundedness, and deficiencies of linear relations and showed that the deficiency of a subspace are stable under bounded perturbation with certain additional conditions ([35], Corollary III. 7.6). The deficiency of a linear relation has a close relationship with its deficiency indices. In 2013, Zheng [36] obtained the invariance of the minimal and maximal deficiency indices under bounded perturbation for discrete Hamiltonian systems. In the present paper, we focus on the study of the invariance of the deficiency indices of (1) under bounded and relatively bounded perturbations. These results obtained in this paper provide an alternate way to determine the limit types of these difference equations, some of which improve and extend some previous results (see Remark 1 and Example 1).

In the study of stability of deficiency indices of equation (1), we encounter a difficulty that the minimal subspaces generated by the unperturbed and perturbed equations are defined in different spaces in the case that a perturbation of weight function occurs. So, it is difficult to study the stability of their deficiency indices by using the results given in [29] (see Lemma 1). In order to overcome this difficulty, we take one transformation to convert the weight function to 1 .

The rest of this paper is organized as follows. In Section 2 , some basic concepts and useful fundamental results about linear relations are introduced. In Section 3, the invariance of deficiency indices of the minimal subspaces corresponding to (1) under bounded and relatively bounded perturbations is built. As a consequence, the invariance of limit types of second-order symmetric linear difference equations under bounded and relatively bounded perturbations is obtained.

\section{Preliminaries}

In this section, some basic concepts and fundamental results about linear relations and second-order symmetric linear difference equations are introduced.

2.1. Some Basic Concepts and Useful Results of Linear Relations. Let $X$ be a complex Hilbert space with inner product $\langle\cdot, \cdot\rangle, T$ and $S$ be two linear relations in the product space $X^{2}:=X \times X$, and $\alpha \in \mathbf{C}$. Denote

$$
\begin{aligned}
D(T) & :=\{x \in X:(x, f) \in T \text { for some } f \in X\}, \\
R(T) & :=\{f \in X:(x, f) \in T \text { for some } x \in X\}, \\
T(x) & :=\{f \in X:(x, f) \in T\}, \\
T^{*} & :=\left\{(y, g) \in X^{2}:\langle g, x\rangle=\langle y, f\rangle \text { for all }(x, f) \in T\right\}, \\
\alpha T & :=\{(x, \alpha f):(x, f) \in T\}, \\
T+S & :=\{(x, f+g):(x, f) \in T,(x, g) \in S\} .
\end{aligned}
$$

A linear relation $T$ is called closed if it is a closed subspace in $X^{2}$. A linear relation $T \subset X^{2}$ is called a Hermitian relation if $T \subset T^{*}$, and it is called a self-adjoint relation if $T=T^{*}$.

Definition 1 (see [17], Definition 2.3). Let $T$ be a linear relation in $X^{2}$. The subspace $R(T-\lambda I)^{\perp}$ is called the deficiency space of $T$ and $\lambda$, and the number $d_{\lambda}(T):=$ $\operatorname{dim} R(T-\lambda I)^{\perp}$ is called the deficiency index of $T$ and $\lambda$.

It can be easily verified that the deficiency indices of $T$ and its closure with the same $\lambda$ are equal. In addition, by [17] (Theorem 2.3), $d_{\lambda}(T)$ is constant in the upper and lower half-planes; that is, $d_{\lambda}(T)=d_{+}(T)$ for all $\lambda \in \mathbf{C}$ with $\operatorname{Im} \lambda>0$ and $d_{\lambda}(T)=d_{-}(T)$, for all $\lambda \in \mathbf{C}$ with $\operatorname{Im} \lambda<0$, 
where $d_{+}(T)=d_{+i}(T)$. The pair $\left(d_{+}(T), d_{-}(T)\right)$ is called the deficiency indices of $T$, and $d_{+}(T)$ and $d_{-}(T)$ are called the positive and negative deficiency indices of $T$, respectively.

We shall recall concepts of the norm of a linear relation and relative boundedness of two linear relations as follows.

Let $T$ be a linear relation in $X^{2}$. The quotient space $X / \overline{T(0)}$ is a Hilbert space [20] with the inner product:

$$
\begin{gathered}
\langle[x],[y]\rangle=\left\langle x^{\perp}, y^{\perp}\right\rangle, \\
{[x],[y] \in X / \overline{T(0)},}
\end{gathered}
$$

where $x=x_{0}+x^{\perp}$ and $y=y_{0}+y^{\perp}$ with $x_{0}, y_{0} \in \overline{T(0)}$ and $x^{\perp}, y^{\perp} \in T(0)^{\perp}$.

Now, define the following natural quotient map:

$$
\mathrm{Q}_{T}: X \longrightarrow X / \overline{T(0)}, \quad x \longmapsto[x] .
$$

Furthermore, we define

$$
\widetilde{T}_{s}=G\left(Q_{T}\right) T,
$$

where $G\left(Q_{T}\right)$ is the graph of $Q_{T}$. Then, $\widetilde{T}_{s}$ is a linear operator with domain $D(T)$ ([35], Proposition II.1.2). The norm of $T$ at $x \in D(T)$ and the norm of $T$ are defined, respectively (see [35], II.1),

$$
\begin{aligned}
\|T(x)\| & :=\left\|\widetilde{T}_{s}(x)\right\|, \\
\|T\| & :=\left\|\widetilde{T}_{s}\right\|=\sup \left\{\left\|\widetilde{T}_{s}(x)\right\|: x \in D(T) \text { with }\|x\| \leq 1\right\} .
\end{aligned}
$$

Definition 2 (see [35], Definition VII.2.1). Let $S$ and $T$ be two linear relations in $X^{2}$.

(1) $S$ is said to be $T$-bounded if $D(T) \subset D(S)$, and there exists a constant $c \geq 0$ such that

$$
\|S(x)\| \leq c(\|x\|+\|T(x)\|), \quad x \in D(T) .
$$

(2) If $S$ is $T$-bounded, then the infimum of all numbers $b \geq 0$ for which a constant $a \geq 0$ exists such that

$$
\|S(x)\| \leq a\|x\|+b\|T(x)\|, \quad x \in D(T),
$$

is called the $T$-bound of $S$.

Lemma 1 (see [29], Corollary 3.1). Let Tand S be Hermitian relations in $X^{2}$ with $D(T) \subset D(S)$ and $S(0) \subset T(0)$. If $S$ is $T$ bounded with $T$-bound less than 1 , then $d_{ \pm}(T+S)=d_{ \pm}(T)$.

2.2. Basic Concepts and Results of Second-Order Symmetric Linear Difference Equations. In this subsection, we introduce the concepts of maximal, preminimal and minimal subspaces, and then list some useful results about (1).

The natural difference operator corresponding to (1) is denoted by

$$
\mathscr{L}(x)(t):=-\nabla(p(t) \Delta x(t))+q(t) x(t), \quad t \in I .
$$

Denote

$$
l_{w}^{2}(0, \infty):=\left\{x=\{x(t)\}_{t=-1}^{\infty} \subset \mathbf{C}: \sum_{t=0}^{\infty} w(t)|x(t)|^{2}<+\infty\right\},
$$

with the inner product $\langle x, y\rangle_{w}:=\sum_{t=0}^{\infty} w(t) \bar{y}(t) x(t)$ and the norm $\|x\|_{w}:=(\langle x, x\rangle)^{1 / 2}$ for any $x, y \in l_{w}^{2}(0, \infty)$. When $w(t) \equiv 1$ for $t \in I, l_{w}^{2}(0, \infty)$ and $\|x\|_{w}$ are denoted by $l^{2}(0, \infty)$ and $\|x\|$, respectively. For convenience, $x$ is called to be square summable if $\|x\| \in l_{w}^{2}(0, \infty)$.

Denote

$$
\begin{aligned}
H & :=\left\{(x, f) \in\left(l_{w}^{2}(I)\right)^{2}: \mathscr{L}(x)(t)=w(t) f(t), \quad t \in I\right\}, \\
H_{00} & :=\left\{(x, f) \in H: \text { there exist two integers } t_{0}, \quad t_{1} \in I \text { with } t_{0}<t_{1} \text { such that } x(t)=0 \text { for } t \leq t_{0} \text { and } t \geq t_{1}\right\},
\end{aligned}
$$

where $H$ and $H_{00}$ are called the maximal and preminimal subspaces corresponding to $\mathscr{L}$ or (1), respectively. The subspace $H_{0}:=\bar{H}_{00}$ is called the minimal subspace corresponding to $\mathscr{L}$ or (1). By Corollary 3.1 and Theorem 3.3 in [10], $H_{00}$ and $H_{0}$ are nondensely defined Hermitian operators in $l_{w}^{2}(0, \infty)$. So, the results about stability of deficiency indices for symmetric operators under perturbations given in [19] cannot be applied to study the invariance of deficiency indices of $H_{0}$ under perturbations. We shall apply the results about invariance of deficiency indices for Hermitian relations under perturbations established in [29] to study this problem.
By $n_{+}\left(H_{0}\right)$ denote the number of linearly independent square summable solutions of (1) with $\lambda= \pm i$ in $l_{w}^{2}(0, \infty)$. By (13) of Lemma 3.5 in [10], we have that $d_{+}\left(H_{0}\right)=$ $n_{ \pm}\left(H_{0}\right)$. In addition, by the largest defect index theorem (see [3], Theorem 5.6.1, or [5], Theorem 3.2.7), either $d_{+}\left(H_{0}\right)=d_{-}\left(H_{0}\right)=1$ or $d_{+}\left(H_{0}\right)=d_{-}\left(H_{0}\right)=2$; that is, $\mathscr{L}$ is either in the limit point type (briefly, l.p.c.) or in the limit circle case (briefly, l.c.c.) at $t=+\infty$.

To the end, we introduce a criterion of limit circle case for (1).

Lemma 2 (see [7], Corollary 3.2). If the coefficients of (1) satisfy 


$$
\begin{aligned}
\delta= & \lim _{t \rightarrow \infty} \sup \left\{\frac{w(t+1)}{w(t-1)}\left(\frac{r(t) r(t-1)}{p(t) p(t-1)}-\frac{p(t-1)}{p(t)}\right)^{2}\right. \\
& +\frac{w(t)}{w(t-1)}\left(\frac{r(t-1)}{p(t-1)}\right)^{2}+\frac{1}{w(t-2)}\left(w(t+1)\left(\frac{r(t)}{p(t)}\right)^{2}\right. \\
& \left.+w(t))\left(\frac{p(t-2)}{p(t-1)}\right)^{2}\right\}<1,
\end{aligned}
$$

where $r(t)=p(t)+p(t-1)+q(t)$ for $t \in I$, then (1) is in l.c.c. at $t=+\infty$.

\section{Main Results}

In this section, we shall study the invariance of deficiency indices of the minimal subspace corresponding to (1) with coefficients under bounded and relatively bounded perturbations. As a consequence, the invariance of limit types of (1) under bounded and relatively bounded perturbations is obtained.

Consider the perturbed difference equation with $p, q$, and $w$ perturbed by $\widetilde{p}, \widetilde{q}$, and $\widetilde{w}$, respectively, i.e.,

$$
\widetilde{\mathscr{L}}(x)(t):=-\nabla(\widetilde{p}(t) \Delta x(t))+\widetilde{q}(t) x(t)=\lambda \widetilde{w}(t) x(t), \quad t \in I,
$$

where $\widetilde{p}, \widetilde{q}$, and $\widetilde{w}$ are real-valued with $\widetilde{p}(t) \neq 0$ for $t \in\{t\}_{t=-1}^{\infty}$ and $\tilde{w}(t)>0$ for $t \in\{t\}_{t=0}^{\infty}$. Similarly, $l_{\widetilde{w}}^{2}(0, \infty),\langle\cdot, \cdot\rangle_{\tilde{w}},\|\cdot\|_{\tilde{w}}$, $\widetilde{H}, \widetilde{H}_{00}, \widetilde{H}_{0}$, and $n_{ \pm}\left(\widetilde{H}_{0}\right)$ are defined as in Section 2.2.

We first consider the case that $w(t)=\widetilde{w}(t) \equiv 1$.

Theorem 1. Assume that $w(t)=\widetilde{w}(t) \equiv 1$ for $t \in I$. If there exists a constant $b$ satisfying $|b|<\sqrt{2} / 2$ such that

$$
\begin{array}{ll}
\widetilde{p}(t)-p(t)=b p(t)+c_{1}(t), & t \geq-1, \\
\widetilde{q}(t)-q(t)=b q(t)+c_{2}(t), & t \geq 0,
\end{array}
$$

where $c_{1}(t)$ and $c_{2}(t)$ are bounded functions of $t$ and $c_{1}(-1)=0$. Then, $d_{ \pm}\left(H_{0}\right)=d_{ \pm}\left(\widetilde{H}_{0}\right)$; that is, $\mathscr{L}$ is in l.c.c. (l.p.c.) at $t=+\infty$ if and only if $\widetilde{\mathscr{L}}$ is in l.c.c. (l.p.c.) at $t=+\infty$.

Proof. By the definition of the preminimal subspace, we have $D\left(H_{00}\right)=D\left(\widetilde{H}_{00}\right)$. Let $S=\widetilde{H}_{00}-H_{00}$. Then, $S$ is Hermitian since $H_{00}$ and $\widetilde{H}_{00}$ are Hermitian. For any $(x, f) \in H_{00}$ and $(x, g) \in S$, we have $(x, f+g) \in \widetilde{H}_{00}$. Thus, $\mathscr{L}(x)(t)=f(t)$ and $\tilde{\mathscr{L}}(x)(t)=f(t)+g(t)$ for $t \in I$. From (14) and that $c_{1}(t)$ and $c_{2}(t)$ are bounded with $c_{1}(-1)=0$, it follows that

$$
\begin{aligned}
\|g\|^{2} & =\|(f+g)-f\|^{2}=\sum_{t=0}^{\infty}|\tilde{\mathscr{L}}(x)(t)-\mathscr{L}(x)(t)|^{2} \\
& =\sum_{t=0}^{\infty}|-\nabla[(\widetilde{p}(t)-p(t)) \Delta x(t)]+(\tilde{q}(t)-q(t)) x(t)|^{2} \\
& =\sum_{t=0}^{\infty}\left|-\nabla\left[\left(b p(t)+c_{1}(t)\right) \Delta x(t)\right]+\left(b q(t)+c_{2}(t)\right) x(t)\right|^{2} \\
& \leq 2 b^{2}\|f\|^{2}+M\|x\|^{2},
\end{aligned}
$$

where $M$ is a positive constant. Therefore, $S$ is $H_{00}$-bounded with $H_{00}$-bound less than 1 since $|b|<\sqrt{2} / 2$. Hence, $d_{ \pm}\left(H_{00}\right)=d_{ \pm}\left(\widetilde{H}_{00}\right)$ by Lemma 1 . This implies that $d_{ \pm}\left(H_{0}\right)=d_{ \pm}\left(\widetilde{H}_{0}\right)$. This completes the proof.

The following result can be directly derived from Theorem 1.

Corollary 1. Assume that $w(t)=\widetilde{w}(t) \equiv 1$ for $t \in I$. If $\{\tilde{p}(t)-p(t)\}_{t=0}^{\infty}$ and $\{\widetilde{q}(t)-q(t)\}_{t=0}^{\infty}$ are bounded, and $|\widetilde{p}(-1)-p(-1)|<(\sqrt{2} / 2)|p(-1)|$, then $d_{ \pm}\left(H_{0}\right)=d_{ \pm}\left(\widetilde{H}_{0}\right)$; that is, $\mathscr{L}$ is in l.c.c. (l.p.c.) at $t=+\infty$ if and only if $\widetilde{\mathscr{L}}$ is in l.c.c. (l.p.c.) at $t=+\infty$.

Remark 1. In [7] (Lemma 2.4), Chen and Shi showed the invariance of the limit point and limit circle cases for (1) with the potential function under bounded perturbation in the case that the weight function $w(t)=\widetilde{w}(t) \equiv 1$ for $t \in I$. Theorem 1 contains this result.

Now, we consider the case that $w(t)=\widetilde{w}(t) \equiv 1$ for $t \in I$. We first introduce the following transform:

$$
\begin{aligned}
& y(t)=w(t)^{1 / 2} x(t), \\
& R(t)=(p(t)+p(t-1)+q(t)) w(t)^{-1}, \\
& P(t)=p(t)(w(t) w(t+1))^{-1 / 2}, \\
& Q(t)=R(t)-P(t)-P(t-1),
\end{aligned}
$$

where $w(-1)$ defined. Applying it, we can convert (1) and (13) to (17) and (18), respectively, that is,

$$
\begin{aligned}
& -\nabla(P(t) \Delta y(t))+Q(t) y(t)=\lambda y(t), \quad t \in I, \\
& -\nabla(\widetilde{P}(t) \Delta y(t))+\widetilde{Q}(t) y(t)=\lambda y(t), \quad t \in I .
\end{aligned}
$$

According to Theorem 1, we get the following result.

Theorem 2. Let $w(t)=\widetilde{w}(t)$ for $t \geq-1$. If there exists $a$ constant $b$ satisfying $|b|<(\sqrt{2} / 2)$ such that

$$
\begin{aligned}
& \widetilde{p}(t)-p(t)= b p(t)+c_{1}(t)(w(t) w(t+1))^{1 / 2}, \quad t \geq-1, \\
& \widetilde{q}(t)-q(t)=b q(t)+c_{2}(t) w(t)-w(t)^{1 / 2} \nabla \\
& \\
& \cdot\left(c_{1}(t)\left(w(t+1)^{1 / 2}-w(t)^{1 / 2}\right)\right), \quad t \geq 0,
\end{aligned}
$$

where $c_{1}(t)$ and $c_{2}(t)$ are bounded functions of $t$ and $c_{1}(-1)=0$. Then, $d_{ \pm}\left(H_{0}\right)=d_{ \pm}\left(\tilde{H}_{0}\right)$; that is, $\mathscr{L}$ is in l.c.c. (l.p.c.) at $t=+\infty$ if and only if $\tilde{\mathscr{L}}$ is in l.c.c. (l.p.c.) at $t=+\infty$.

Corollary 2. Let $w(t)=\widetilde{w}(t)$ for $t \geq-1$. If $\left\{(\widetilde{p}(t)-p(t))(w(t) w(t+1))^{-1 / 2}\right\}_{t=0}^{\infty}$ and $\{(\widetilde{p}(t)+\widetilde{p}(t-$ 1) $\left.+\widetilde{q}(t)-p(t)-p(t-1)-q(t)) w(t)^{-1}\right\}_{t=0}^{\infty}$ are bounded, and $|\widetilde{p}(-1)-p(-1)|<(\sqrt{2} / 2)|p(-1)|$, then $d_{ \pm}\left(H_{0}\right)=$ $d_{ \pm}\left(\widetilde{H}_{0}\right)$; that is, $\mathscr{L}$ is in l.c.c. (l.p.c.) at $t=+\infty$ if and only if $\widetilde{\mathscr{L}}$ is in l.c.c. (l.p.c.) at $t=+\infty$.

Next, we shall consider the case that $p(t)=\widetilde{p}(t)$ for $t \in\{t\}_{t=-1}^{\infty}$ and $q(t)=\widetilde{q}(t)$ for $t \in I$.

Lemma 3. Let $p(t)=\widetilde{p}(t)$ for $t \in\{t\}_{t=-1}^{\infty}$ and $q(t)=\widetilde{q}(t)$ for $t \in I$. If there exists a constant $\alpha>0$ and $t_{0} \in I$ such that 


$$
\widetilde{w}(t) \leq \alpha w(t), \quad t \geq t_{0},
$$

then $d_{ \pm}\left(H_{0}\right) \leq d_{ \pm}\left(\tilde{H}_{0}\right)$.

Proof. In view of $p(t)=\widetilde{p}(t)$ for $t \in\{t\}_{t=-1}^{\infty}$ and $q(t)=\widetilde{q}(t)$ for $t \in I$, it follows that (1) and (13) with $\lambda=0$ have the same solutions. On the contrary, by (20), we have that $l_{w}^{2}(0, \infty) \subset l_{w}^{2}(0, \infty)$. Therefore, it follows from the maximal defect index theorem (see [3], Theorem 5.6.1, or [5], Theorem 3.2.7) that $d_{ \pm}\left(H_{0}\right) \leq d_{ \pm}\left(\widetilde{H}_{0}\right)$. This completes the proof.

The following result can be directly derived from Lemma 3.

Theorem 3. Let $p(t)=\widetilde{p}(t)$ for $t \in\{t\}_{t=-1}^{\infty}$ and $q(t)=\widetilde{q}(t)$ for $t \in I$. If there exist constants $\alpha, \beta>0$, and $t_{0} \in I$ such that

$$
\beta w(t) \leq \tilde{w}(t) \leq \alpha w(t), \quad t \geq t_{0},
$$

then $d_{ \pm}\left(H_{0}\right)=d_{ \pm}\left(\tilde{H}_{0}\right)$.

Combing Theorems 2 and 3, we can get the following result.

Theorem 4. If (19) and (21) hold, then $d_{ \pm}\left(H_{0}\right)=d_{ \pm}\left(\widetilde{H}_{0}\right)$; that is, $\mathscr{L}$ is in l.c.c. (l.p.c.) at $t=+\infty$ if and only if $\widetilde{\mathscr{L}}$ is in l.c.c. (l.p.c.) at $t=+\infty$.

To conclude this section, we shall give an example to illustrate the results obtained in this paper.

Example 1. Let $p(t)=4^{t}$ for $t \geq-1, q(t)=-4^{t}$ and $w(t)=$ $t+1$ for $t \geq 0$ in (1). Then,

$$
\mathscr{L}(x)(t)=-\nabla\left(4^{t} \Delta x(t)\right)-4^{t} x(t)=\lambda(t+1) x(t), \quad t \in I,
$$

is in l.c.c. at $t=+\infty$ by Lemma 2 with $\delta=(21 / 128)<1$. This implies that $d_{ \pm}\left(H_{0}\right)=2$.

Consider the perturbed difference equation:

$$
\widetilde{\mathscr{L}}(x)(t):=-\nabla(\widetilde{p}(t) \Delta x(t))+\widetilde{q}(t) x(t)=\lambda \widetilde{w}(t) x(t), \quad t \in I,
$$

with $\widetilde{p}(-1)=1 / 8$,

$$
\begin{aligned}
& \widetilde{p}(t)=2 \cdot 4^{t-1}+\sqrt{(t+1)(t+2)}, \\
& \widetilde{q}(t)=-2 \cdot 4^{t-1}+3 t+3-\sqrt{(t+1)(t+2)}-\sqrt{t(t+1)}, \\
& \widetilde{w}(t)=5 t+3, \quad t \geq 0 .
\end{aligned}
$$

In order to avoid complicated calculation for (12), it is convenient to treat the perturbed equation by using Theorem 4 instead of Lemma 2 . It can be easily verified that the conditions in Theorem 4 are satisfied with $b=-1 / 2$, $c_{1}(t)=c_{2}(t)=1$, for $t \geq 0, \alpha=5$, and $\beta=1$. Thus, it follows from Theorem 4 that $d_{ \pm}\left(\tilde{H}_{0}\right)=d_{ \pm}\left(H_{0}\right)=2$. Therefore, $\widetilde{\mathscr{L}}$ is also in l.c.c. at $t=+\infty$.

\section{Conclusions}

As we have mentioned in the first section, it is very important for us to study the stability of deficiency indices of both differential equations and difference equations because the deficiency indices play an important role in theory of self-adjoint extension.

This paper is concerned with stability of deficiency indices of second-order symmetric linear difference equations with one singular endpoint under bounded and relatively bounded perturbations. By applying the perturbation theory of Hermitian linear relations, several criteria of stability of deficiency indices of second-order symmetric linear difference equations are established. These results obtained in this paper provide an alternate way to determine the limit types of these difference equations, some of which improve and extend some previous results (see Remark 1 and Example 1). In addition, since the deficiency index equals to the number of linearly independent square summable solutions of (1) with $\lambda \in \mathbf{C} / \mathbf{R}$, the results given in this paper may also be used to determine the number of linearly independent square summable solutions. These results obtained in the present paper will be a foundation of research on stability of spectra of these difference equations. Finally, we shall remark that the ideas and methods used in this paper are still available in the study of invariance of the deficiency indices of discrete Hamiltonian systems. And, we shall discuss this problem in the near future.

\section{Data Availability}

No data were used to support this study.

\section{Conflicts of Interest}

The author declares no conflicts of interest regarding the publication of this paper.

\section{Authors' Contributions}

The author carried out the proof and conceived the study. The author read and approved the final manuscript.

\section{Acknowledgments}

The author would like to thank the referee for carefully reading her manuscript and giving some valuable suggestions. This research was supported by the NNSF of China (Grant no. 11901153) and NSF of Jiangsu Province (Grant no. BK20170298).

\section{References}

[1] Y. Last and B. Simon, "Eigenfunctions, transfer matrices, and absolutely continuous spectrum of one-dimensional Schrödinger operators," Inventiones Mathematicae, vol. 135, no. 2, pp. 329-367, 1999.

[2] C. Remling, "The absolutely continuous spectrum of onedimensional schrödinger operators with decaying potentials," Communications in Mathematical Physics, vol. 193, no. 1, pp. 151-170, 1998. 
[3] F. V. Atkinson, Discrete and Continuous Boundary Problems, Academic Press, New York, NY, USA, 1964.

[4] D. B. Hinton and R. T. Lewis, "Spectral analysis of second order difference equations," Journal of Mathematical Analysis and Applications, vol. 63, no. 2, pp. 421-438, 1978.

[5] A. Jirari, "Second-order Sturm-Liouville difference equations and orthogonal polynomials," Memoirs of the American Mathematical Society, vol. 113, no. 542, 1995.

[6] S. L. Clark, "A spectral analysis for self-adjoint operators generated by a class of second order difference equations," Journal of Mathematical Analysis and Applications, vol. 197, no. 1, pp. 267-285, 1996.

[7] J. Chen and Y. Shi, "The limit circle and limit point criteria for second-order linear difference equations," Computers \& Mathematics with Applications, vol. 47, no. 6-7, pp. 967-976, 2004.

[8] H. Sun and Y. Shi, "Eigenvalues of second-order difference equations with coupled boundary conditions," Linear Algebra and Its Applications, vol. 414, no. 1, pp. 361-372, 2006.

[9] Y. Wang and Y. Shi, "Eigenvalues of second-order difference equations with periodic and antiperiodic boundary conditions," Journal of Mathematical Analysis and Applications, vol. 309, no. 1, pp. 56-69, 2005.

[10] Y. Shi and H. Sun, "Self-adjoint extensions for second-order symmetric linear difference equations," Linear Algebra and Its Applications, vol. 434, no. 4, pp. 903-930, 2011.

[11] Y. Liu and Y. Shi, "Regular approximations of spectra of singular second-order symmetric linear difference equations," Linear Algebra and Its Applications, vol. 444, pp. 183-210, 2014.

[12] Y. Liu and Y. Shi, "Regular approximations of isolated eigenvalues of singular second-order symmetric linear difference equations," Advances in Difference Equations, vol. 2016, no. 1, 2016.

[13] Y. Liu and Y. Shi, "Approximation of eigenvalues below the essential spectra of singular second-order symmetric linear difference equations," Science China Mathematics, vol. 60, no. 9, pp. 1661-1678, 2017.

[14] R. Hilscher, "Discrete spectra criteria for certain classes of singular differential and difference operators," Computers \& Mathematics with Applications, vol. 42, no. 3-5, pp. 465-476, 2001.

[15] S. Peňa, "Discrete spectral criteria for singular difference operators," Mathematica Bohemica, vol. 124, pp. 35-44, 1999.

[16] E. A. Coddington, "Extension theory of formally normal and symmetric subspaces," Memoirs of the American Mathematical Society, no. 134, 1973.

[17] Y. Shi, "The Glazman-Krein-Naimark theory for Hermitian subspaces," Journal of Operator Theory, vol. 68, pp. 241-256, 2012.

[18] H. Weyl, "Über gewöhnliche Differentialgleichungen mit Singularitäten und die zugehörigen Entwicklungen willkürlicher Funktionen," Mathematische Annalen, vol. 68, no. 2, pp. 220-269, 1910.

[19] H. Behncke and H. Focke, "Stability of deficiency indices," Proceedings of the Royal Society of Edinburgh: Section A Mathematics, vol. 78, no. 1-2, pp. 119-127, 1977.

[20] T. Kato, Perturbation Theory for Linear Operators, Springer, Berlin, Germany, 2nd edition, 1984.

[21] A. Devinatz, "The deficiency index problem for ordinary self adjoint differential operators," Bulletin of the American Mathematical Society, vol. 79, no. 6, pp. 1109-1128, 1973.

[22] E. Kissin, "Stability of the deficiency indices of symmetric operators under self-adjoint perturbations," Proceedings of the
Edinburgh Mathematical Society, vol. 46, no. 2, pp. 383-394, 2003.

[23] A. Zettl, "Perturbation theory of deficiency indices of differential operators," Journal of the London Mathematical Society, vol. s2-12, no. 4, pp. 405-412, 1976.

[24] J. O. Agure, D. O. Ambogo, and F. O. Nyamwala, "Deficiency indices and spectrum of fourth order difference equations with unbounded coefficients," Mathematische Nachrichten, vol. 286, no. 4, pp. 323-339, 2013.

[25] H. Behncke, "Spectral theory of Hamiltonian difference systems with almost constant coefficients," Journal of Difference Equations and Applications, vol. 19, no. 1, pp. 1-12, 2013.

[26] Y. Shi, "Weyl-Titchmarsh theory for a class of discrete linear Hamiltonian systems," Linear Algebra and Its Applications, vol. 416, no. 2-3, pp. 452-519, 2006.

[27] J. V. Neumann, Functional Operators II: The Geometry of Orthogonal Spaces, Princeton University Press, Princeton, NJ, USA, 1950.

[28] R. Arens, "Operational calculus of linear relations," Pacific Journal of Mathematics, vol. 11, no. 1, pp. 9-23, 1961.

[29] Y. Liu and Y. Shi, "Invariance of deficiency indices of Hermitian subspaces under relatively bounded perturbations (preprint)," 2019, http://arxiv.org/pdf/1904.06011.

[30] Y. Liu, "On the stability of self-adjointness of linear relations," Discrete Dynamics in Nature and Society, vol. 2019, Article ID 6784546, 5 pages, 2019.

[31] S. Clark and F. Gesztesy, "On Weyl-Titchmarsh theory for singular finite difference Hamiltonian systems," Journal of Computational and Applied Mathematics, vol. 171, no. 1-2, pp. 151-184, 2004.

[32] G. Ren and Y. Shi, "The defect index of singular symmetric linear difference equations with real coefficients," Proceedings of the American Mathematical Society, vol. 138, no. 07, p. 2463, 2010.

[33] G. Ren and Y. Shi, "Defect indices and definiteness conditions for a class of discrete linear Hamiltonian systems," Applied Mathematics and Computation, vol. 218, no. 7, pp. 3414-3429, 2011.

[34] Y. Shi, "Symplectic structure of discrete Hamiltonian systems," Journal of Mathematical Analysis and Applications, vol. 266, no. 2, pp. 472-478, 2002.

[35] R. Cross, "Multivalued linear operators," in Monographs and Textbooks in Pure and Applied Mathematics, vol. 213, Marcel Dekker, New York, NY, USA, 1998.

[36] Z. Zheng, "Invariance of deficiency indices under perturbation for discrete Hamiltonian systems," Journal of Difference Equations and Applications, vol. 19, no. 8, pp. 1243-1250, 2013. 